\title{
Inferior Mean of Measures on Curves and Subspaces
}

\section{Yevgenya Movshovich}

Eastern Illinois University, Charleston, IL 61920, USA

Correspondence should be addressed to Yevgenya Movshovich; ymovshovich@eiu.edu

Received 31 May 2014; Revised 2 September 2014; Accepted 3 September 2014; Published 28 December 2014

Academic Editor: Mohsen Tadi

Copyright (C) 2014 Yevgenya Movshovich. This is an open access article distributed under the Creative Commons Attribution License, which permits unrestricted use, distribution, and reproduction in any medium, provided the original work is properly cited.

We obtain new sharp upper bounds of the inferior mean for positive harmonic functions defined by finite boundary measures that lie on curves or subspaces of the boundary of the half-space.

"Dedicated to L. D. Ivanov and my mother, Faina M. Movshovich"

\section{Introduction}

Let $\Omega$ be the unit ball, $S^{n}$, or the upper half-space, $R_{+}^{n+1}$, in $R^{n+1}$, and let $\partial \Omega$ be the boundary of $\Omega$. The notion of the inferior mean, $\operatorname{IM}(u)$, of a positive function $u$ is due to Maurice Heins.

Definition 1. Let $u$ be a positive function defined on $\Omega$ and let $A_{\delta}=\{q \in \Omega: \operatorname{dist}(q, \partial \Omega)<\delta\}$. The inferior mean of $u$ is

$$
\operatorname{IM}(u)=\lim _{\delta \rightarrow 0} \inf _{\Gamma \subset A_{\delta}} \int_{\Gamma} u(q) d \Gamma
$$

where $d \Gamma$ is the volume measure of a continuous, piecewise differentiable, orientable surface $\Gamma$ that separates boundaries of $A_{\delta}$.

The study of the inferior mean began with the following unpublished result of Heins, which is obtained in connection with $E_{P}$ spaces.

If a positive function $u$ has a subharmonic logarithm on the annulus $\{R<|z|<1\}$ and $\Gamma$ are rectifiable Jordan curves in $\{r<|z|<1\}$ separating 0 from $\infty$, then

$$
\liminf _{r \rightarrow 1} \int_{\Gamma} u(z)|d z|=\lim _{r \rightarrow 1} \int_{|z|=r} u(z)|d z| .
$$

In [1], $\mathrm{Wu}$ showed that, for a positive harmonic function $u$ defined on the unit disc, the same result holds, if the boundary measure $\mu$ of $u$ is absolutely continuous with respect to the arclength measure of the unit circle; while, for an arbitrary positive harmonic function $u$ in the disc, $(2 / \pi) \mu(\partial \Omega) \leq \operatorname{IM}(u) \leq \mu(\partial \Omega)$. In [2], these results of Wu were extended to positive functions $u$ in the harmonic Hardy space $h^{1}(\Omega)$, which is the space of harmonic functions defined on $\Omega$ by finite (positive) Borel boundary measures $\mu$ :

$$
\frac{\omega_{n+1}}{\pi \omega_{n}} \mu(\partial \Omega) \leq \mathrm{IM}(u) \leq \mu(\partial \Omega),
$$

where $\omega_{k}$ is the area of the unit sphere $S^{k}$ in $R^{k+1}$. Equality on the right holds for functions whose boundary measures are absolutely continuous with respect to the Lebesgue measure of $\partial \Omega$.

This paper refines the upper bound in (3) for $u \in h^{1}(\Omega)$ defined on the upper half-space $\Omega=R_{+}^{n+1}$ by a finite positive Borel boundary measure $\mu$ that lies either on a subspace or on a smooth curve $\gamma \subset \partial \Omega$. With $\mu(\gamma)=\mu(\partial \Omega)$, we will prove the following theorems (throughout smooth means $C^{k}$ differentiable, $k \geq 1$ ).

In Theorem 5 we show that if $\mu$ lies on a subspace $\gamma=R^{m}$, $m \in\{0, \ldots, n\}$, then

$$
\operatorname{IM}(u) \leq \frac{\omega_{n-m+1}}{\pi \omega_{n-m}} \mu(\gamma)
$$


In Theorem 7 we show that equality in (4) holds for boundary measures $\mu$ that are absolutely continuous with respect to the Lebesgue measure of $\gamma$.

In Theorem 9 we show that if $\mu$ lies on a smooth curve $\gamma$, then (4) is true with $m=1$.

In Theorem 10 we show that equality in (4) holds for the boundary measure $\mu$ that is the arclength measure of $\gamma$.

\section{Tubular Coordinates}

In this section, we recall a few facts about spherical transformations, obtain the volume elements of hypersurfaces in tubular coordinates, and evaluate three constants occurring in the integration over the tubes. In all sections, depending on the context, we use the same notation for points and position vectors, vectors and line segments, and so forth.

2.1. Spherical Coordinates. Let $q \in R_{+}^{k+1}=R_{+} \times R^{k}$. In Cartesian coordinates $q=\left(x_{0}, \mathbf{x}\right)$, one has $x_{0} \in R_{+}$and $\mathbf{x}=\left(x_{1}\right.$, $\left.\ldots, x_{k}\right) \in R^{k}$. In local spherical coordinates with the origin at $p \in R^{k}$, spherical radius $r=|q-p|$, the first spherical angle $\phi_{1} \in[0, \pi / 2)$ is measured from $R_{+}$to $q-p$, and the remaining spherical angles lie in $R^{k}$ with the range: $\phi_{i} \in[0, \pi], 1<i<k$, $\phi_{k} \in[0,2 \pi]$. A $k$-tuple $\left(\phi_{1}, \ldots, \phi_{k}\right)$ will be denoted by $\phi$.

Define $\Lambda_{i}$ by $\Lambda_{0}=1, \Lambda_{i}=\prod_{j=1}^{i} \sin \phi_{j}$. The spherical transformation from Cartesian to spherical coordinates is given by the identities:

$$
x_{i}=r \Lambda_{i} \cos \phi_{i+1}, \quad i=0, \ldots, k-1, \quad x_{k}=r \Lambda_{k} .
$$

We denote by $S^{k}$ the unit sphere in $R^{k+1}$ and by $S_{+}^{k}=S^{k} \cap$ $R_{+}^{k+1}$ its upper-half in $R^{k+1}$. The volume element of a sphere of constant radius $r$ (see e.g., [3, Section 676]) is

$$
r^{k} d S^{k}=I_{k} d \phi_{1} \cdots d \phi_{k}
$$

where

$$
I_{k}=\frac{D\left(x_{0}, \ldots, x_{k}\right)}{D\left(r, \phi_{1}, \ldots, \phi_{k}\right)}=\frac{D\left(x_{0}, \mathbf{x}\right)}{D(r, \phi)}=r^{k} \prod_{i=0}^{k} \Lambda_{i} .
$$

We recall recurrence relations for the area, $\omega_{k}$, and area element of $S^{k}$ :

$$
\begin{aligned}
& \omega_{k+1}=\frac{2 \pi}{k} \omega_{k-1}, \quad \omega_{0}=2, \quad \omega_{1}=2 \pi, \\
& d S^{k}\left(\phi_{1}, \ldots, \phi_{k}\right) \\
& =\sin ^{k-1} \phi_{1} d \phi_{1} d S^{k-1}\left(\phi_{2}, \ldots, \phi_{k}\right) .
\end{aligned}
$$

2.2. Tubular (Cylindrical) Coordinates. In tubular coordinates, points $q=(r, \boldsymbol{\phi}, \mathbf{z})$ have $(r, \phi)=\left(x_{0}, \mathbf{x}\right) \in R_{+}^{n-m+1}$ with $r=\operatorname{dist}\left(q, R^{m}\right), \phi=\left(\phi_{1}, \ldots, \phi_{n-m}\right)$, and $\mathbf{z} \in R^{m}$. The volume element of a tubular surface $T_{r}^{m}$ about $R^{m}$ of constant radius $r$ is given by (6) and (7):

$$
d T_{r}^{m}(q)=r^{n-m} d S^{n-m}(\phi) d \mathbf{z}=I_{n-m} d \phi d \mathbf{z} .
$$

The next result is a corollary to the surface measure lemma in [2].
Lemma 2. If a hypersurface $\Gamma$ is locally given by a $C^{1}$ differentiable function $r=r(\phi, \mathbf{z})$, then its volume element admits this form:

$$
d \Gamma(q)=\sqrt{1+\sum_{k=1}^{n-m} \frac{r_{\phi_{k}}^{2}}{r^{2} \Lambda_{k-1}^{2}}+\sum_{k=1}^{m} r_{z_{k}}^{2}} d T_{r}^{m}(q)
$$

Proof. By Cramer's rule (see e.g., [2]),

$$
\begin{aligned}
d \Gamma= & \left(\sum_{k=0}^{n-m}\left|\frac{D\left(\ldots, \widehat{x_{k}}, \ldots, \mathbf{z}\right)}{D(\phi, \mathbf{z})}\right|^{2}\right. \\
& \left.+\sum_{k=1}^{m}\left|\frac{D\left(x_{0}, \mathbf{x}, \ldots, \widehat{z_{k}}, \ldots\right)}{D(\phi, \mathbf{z})}\right|^{2}\right)^{1 / 2} d \phi d \mathbf{z},
\end{aligned}
$$

where $\widehat{x_{k}}$ and $\widehat{z_{k}}$ indicate that columns

$$
\begin{gathered}
\left(\frac{\partial x_{k}}{\partial \phi_{1}}, \ldots, \frac{\partial x_{k}}{\partial \phi_{n-m}}, \frac{\partial x_{k}}{\partial z_{1}}, \ldots, \frac{\partial x_{k}}{\partial z_{m}}\right) \\
\left(\frac{\partial z_{k}}{\partial \phi_{1}}, \ldots, \frac{\partial z_{k}}{\partial \phi_{n-m}}, \frac{\partial z_{k}}{\partial z_{1}}, \ldots, \frac{\partial z_{k}}{\partial z_{m}}\right) \\
=\left(0, \ldots, 0, \frac{\partial z_{k}}{\partial z_{k}}, 0, \ldots, 0\right)
\end{gathered}
$$

are omitted. The surface measure lemma in [2] computes the first sum in (12) as

$$
\begin{aligned}
\sum_{k=0}^{n-m}\left|\frac{D\left(\ldots, \widehat{x_{k}}, \ldots, \mathbf{z}\right)}{D(\boldsymbol{\phi}, \mathbf{z})}\right|^{2} & =\sum_{k=0}^{n-m}\left|\frac{D\left(\ldots, \widehat{x_{k}}, \ldots\right)}{D(\phi)}\right|^{2} \\
& =I_{n-m}^{2}\left(1+\sum_{k=1}^{n-m} \frac{r_{\phi_{k}}^{2}}{r^{2} \Lambda_{k-1}^{2}}\right) .
\end{aligned}
$$

When the chain rule is applied to each Jacobian in the second sum of (12), the definition (7) for $I_{n-m}$ allows us to write this sum as

$$
\begin{aligned}
\sum_{k=1}^{m}\left|\frac{D\left(x_{0}, \mathbf{x}, \ldots, \widehat{z_{k}}, \ldots\right)}{D(\boldsymbol{\phi}, \mathbf{z})}\right|^{2} & =\sum_{k=1}^{m}\left|\frac{D\left(x_{0}, \mathbf{x}\right)}{D(r, \boldsymbol{\phi})} \frac{D(r, \boldsymbol{\phi})}{D\left(\boldsymbol{\phi}, z_{k}\right)}\right|^{2} \\
& =I_{n-m}^{2} \sum_{k=1}^{m} r_{z_{k}}^{2} .
\end{aligned}
$$

2.3. A Remark on Integration in Tubular Coordinates. For $\delta>$ 0 , let $A_{\delta}=\left\{q \in R_{+}^{n+1}: \operatorname{dist}\left(q, R^{n}\right)<\delta\right\}$, where $R^{n}$ is the boundary of $R_{+}^{n+1}$. Consider a continuous, piecewise smooth, orientable hypersurface $\Gamma \subset A_{\delta}$ separating boundaries of $A_{\delta}$. We assume that each smooth component of $\Gamma$ has a piecewise smooth boundary. Denote by $\Psi$ the following set:

$$
\begin{aligned}
\Psi=\left\{(\boldsymbol{\phi}, \mathbf{z}): \phi_{1} \in\left[0, \frac{\pi}{2}\right), \phi_{i} \in[0, \pi],\right. \\
\left.\quad 1<i<n-m, \phi_{n-m} \in[0,2 \pi], \mathbf{z} \in R^{m}\right\} .
\end{aligned}
$$


Then, $\Psi$ is a subspace of coordinate space $(r, \boldsymbol{\phi}, \mathbf{z})$ in $R_{+}^{n+1}$ and can be regarded as an $n$-dim manifold without boundary. A projection $\Gamma \rightarrow \Psi$ given by $(r, \boldsymbol{\phi}, \mathbf{z}) \rightarrow(1, \boldsymbol{\phi}, \mathbf{z}) \rightarrow(\phi, \mathbf{z})$ is continuous, onto, and smooth on each smooth component of $\Gamma$. Under this projection by Sard's theorem [4], the image of points $q \in \Gamma$, where $r(\phi, \mathbf{z})$ is defined in some neighborhood of $q$, has full measure in $\Psi$. This will justify integration over $\Gamma$ in coordinates $(r(\boldsymbol{\phi}, \mathbf{z}), \boldsymbol{\phi}, \mathbf{z})$ in Theorems 7 and 10.

2.4. Constants $g_{m, n}, G_{m, n}$, and $S_{m, n}$. The first set, $\left\{g_{m, n}\right\}$, is defined for $m=1, \ldots, n$. These are Eulerian integrals (see e.g., [3, Section 534]):

$$
\begin{aligned}
g_{m, n} & =\int_{0}^{\infty} g(v) d v=\int_{0}^{\infty} \frac{v^{m-1} d v}{\left(1+v^{2}\right)^{(n+1) / 2}} \\
& =\frac{\omega_{n}}{\omega_{m-1} \omega_{n-m}} .
\end{aligned}
$$

The second set, $\left\{G_{m, n}\right\}$, is defined for $m=0, \ldots, n$, where $G_{0, n}=1$ and

$$
\begin{aligned}
G_{m, n} & =\int_{R^{m}} \frac{d \mathbf{v}}{\left(1+|\mathbf{v}|^{2}\right)^{(n+1) / 2}}=\omega_{m-1} g_{m, n} \\
& =\frac{\omega_{n}}{\omega_{n-m}}, \quad(m \geq 1) .
\end{aligned}
$$

The next lemma is used in Theorems 7 and 10 with $\Upsilon$ small or $\emptyset$.

Lemma 3. Let $\rho>0, \Upsilon \subset R^{m}$, and $B(0, \rho)=\left\{\mathbf{v} \in R^{m}:|\mathbf{v}| \leq\right.$ $\rho\}$. Then,

$$
\begin{array}{r}
\int_{B(0, \rho) \backslash Y} \frac{d \mathbf{v}}{\left(1+|\mathbf{v}|^{2}\right)^{(n+1) / 2}}>G_{m, n}-\frac{34}{\rho}-|Y|, \\
m=1, \ldots, n .
\end{array}
$$

Proof. We split the integral into three parts and then use $\int_{\rho}^{\infty} g(v) d v \leq 1 / \rho$ and $\max _{k} \omega_{k}=\omega_{6}<34$ in the middle integral:

$$
\begin{aligned}
& \int_{R^{m}} \frac{d \mathbf{v}}{\left(1+|\mathbf{v}|^{2}\right)^{(n+1) / 2}}-\int_{R^{m} \backslash B(0, \rho)} \frac{d \mathbf{v}}{\left(1+|\mathbf{v}|^{2}\right)^{(n+1) / 2}} \\
& -\int_{B(0, \rho) \cap \Upsilon} \frac{d \mathbf{v}}{\left(1+|\mathbf{v}|^{2}\right)^{(n+1) / 2}} .
\end{aligned}
$$

The third set, $\left\{S_{m, n}\right\}$, is also defined for $m=0, \ldots, n$. These are the integrals of $\cos \phi_{1}$ over $S_{+}^{k}$ (see (22) below). In the halfspace $\Omega=R_{+}^{n+1}$, it is customary to denote $\operatorname{dist}(q, \partial \Omega)$ by $y$. If $q=(y, \mathbf{x}, \mathbf{z})=(r, \boldsymbol{\phi}, \mathbf{z})$ is given in tubular coordinates of Section 2.2, then

$$
\begin{gathered}
r=\operatorname{dist}\left(q, R^{m}\right)=\sqrt{y^{2}+|\mathbf{x}|^{2}}, \\
\text { where } y= \begin{cases}r \cos \phi_{1}, & m<n, \\
r, & m=n .\end{cases}
\end{gathered}
$$

With the help of (9) and (8), we evaluate the constants:

$$
S_{m, n}=\int_{S_{+}^{n-m}} \frac{y}{r} d S^{n-m}(\phi)=\frac{\omega_{n-m+1}}{2 \pi} .
$$

\section{Poisson Kernel, Invariants, and Herglotz Representation of Harmonic Functions}

3.1. Poisson Integral Invariants $P_{m, n}$. The Poisson kernel, $P(q$, $p)$, is a function of $q=(y, \mathbf{x}, \mathbf{z}) \in \Omega=R_{+}^{n+1}$ and $p \in \partial \Omega=R^{n}$ :

$$
P(q, p)=\frac{2 \operatorname{dist}(q, \partial \Omega)}{\omega_{n}|q-p|^{n+1}}=\frac{2 y}{\omega_{n}|q-p|^{n+1}} .
$$

Lemma 4. Let $m \in\{0, \ldots, n\}$. The integrals of $P$ over halftubes $T_{r}^{m} \cap \Omega$ do not depend on $r$, denoted by $P_{m, n}$; they are evaluated as

$$
\begin{aligned}
P_{m, n} & =\int_{T_{r}^{m} \cap \Omega} P(q, p) d T_{r}^{m}(q)=\frac{2}{\omega_{n}} S_{m, n} G_{m, n} \\
& =\frac{\omega_{n-m+1}}{\pi \omega_{n-m}} .
\end{aligned}
$$

Proof. Let $q=(r, \boldsymbol{\phi}, \mathbf{z})=(y, \mathbf{x}, \mathbf{z}) \in R_{+} \times R^{n-m} \times R^{m}$ and $p=\left(0, \mathbf{0}, \mathbf{z}_{p}\right)$. Define $\mathbf{v}=\left(\mathbf{z}-\mathbf{z}_{p}\right) / r$. Then, (21) implies that $|q-p|^{2}=r^{2}\left(1+|\mathbf{v}|^{2}\right)$.

The invariants are computed with the help of (10), (18), and (22):

$$
\begin{aligned}
P_{m, n}= & \int_{T_{r}^{m} \cap \Omega} \frac{2 y d T_{r}^{m}(q)}{\omega_{n}|q-p|^{n+1}} \\
= & \int_{R^{m}} \int_{S_{+}^{n-m}} \frac{2 y r^{n-m} d S^{n-m}(\phi) d \mathbf{z}}{\omega_{n}\left[r^{2}+\left|\mathbf{z}-\mathbf{z}_{p}\right|^{2}\right]^{(n+1) / 2}} \\
= & \frac{2}{\omega_{n}} \int_{S_{+}^{n-m}} \frac{y}{r} d S^{n-m}(\phi) \\
& \cdot \int_{R^{m}} \frac{d \mathbf{v}}{\left(1+|\mathbf{v}|^{2}\right)^{(n+1) / 2}}=\frac{2}{\omega_{n}} S_{m, n} G_{m, n} .
\end{aligned}
$$

Herglotz Representation (see [5-7]). A positive harmonic function $u \in h^{1}(\Omega)$ is defined by a finite positive Borel measure $\mu$ on $\partial \Omega$. It can be expressed as the Poisson-Stieltjes integral of $\mu$ :

$$
u(q)=\int_{\partial \Omega} P(q, p) d \mu(p)=\int_{R^{n}} \frac{2 y}{\omega_{n}|q-p|^{n+1}} d \mu(p) .
$$

3.2. Integration over Hyperplanes with Holes. Let $p_{0} \in \partial \Omega$ and $H=\left\{q=(y, \mathbf{x}, \mathbf{z}): y=r^{2}\right\}, 0<r<1$. Then, (see e.g., [2, Section 5])

$$
\int_{H \cap\left\{\left|q-p_{0}\right|>r\right\}} P\left(q, p_{0}\right) d H(q)=O(r) .
$$


Now, consider a subset of $H$ with holes above the support of $\mu$ :

$$
\begin{array}{r}
H_{r}^{\mu}=\left\{q \in \Omega: y=r^{2}, \operatorname{dist}(q, \operatorname{supp}(\mu)) \geq r\right\}, \\
0<r<1 .
\end{array}
$$

Fubini's Theorem and (27) yield

$$
\int_{H_{r}^{\mu}} u d H=O(r) \mu(\partial \Omega)=O(r) .
$$

\section{Measures on Subspaces}

Here, we show that if $u$ is defined by a boundary measure $\mu$ lying on a subspace $R^{m}$, then $\operatorname{IM}(u)$ is bounded by the integrals of $u$ over the half-tubes $T_{r}^{m} \cap \Omega$. If $\mu$ is absolutely continuous with respect to the volume measure of $R^{m}$, these integrals approach $\operatorname{IM}(u)$ as $r \rightarrow 0$.

\subsection{Inequality (4) for $\mu$ on $R^{m}$}

Theorem 5. Let $\Omega$ be the upper half-space $R_{+}^{n+1}$. Let a harmonic function $u$ be defined on $\Omega$ by a finite positive Borel boundary measure $\mu$ that lies on a subspace $R^{m} \subset \partial \Omega$. Then

$$
I M(u) \leq P_{m, n} \mu\left(R^{m}\right) .
$$

Proof. Fix $\delta>0$ and let $r<\delta$. Consider hypersurfaces $\Gamma_{r}=\Gamma_{1} \cup \Gamma_{2}$ separating the two boundaries of $A_{\delta}=\{q \in$ $\Omega: \operatorname{dist}(q, \partial \Omega)<\delta\}$ :

$$
\begin{aligned}
& \Gamma_{1}=\left\{q \in \Omega: \quad y=r^{2}, \operatorname{dist}\left(q, R^{m}\right)>r\right\}, \\
& \Gamma_{2}=\left\{q \in \Omega: y \geq r^{2}, \operatorname{dist}\left(q, R^{m}\right)=r\right\} .
\end{aligned}
$$

Then, $\Gamma_{1} \subset H_{r}^{\mu}$ defined by (28) and $\Gamma_{2} \subset T_{r}^{m}$ defined in Section 2.2. Now, (30) follows from (1), Fubini's Theorem, Lemma 4, and (29):

$$
\begin{aligned}
\operatorname{IM}(u) & \leq \lim _{r \rightarrow 0}\left(\int_{T_{r}^{m} \cap \Omega} u(q) d T_{r}^{m}(q)+\int_{H_{r}^{\mu}} u(q) d H(q)\right) \\
& =\lim _{r \rightarrow 0}\left(\int_{\partial \Omega} \int_{T_{r}^{m} \cap \Omega} P(q, p) d T_{r}^{m}(q) d \mu(p)+O(r)\right) \\
& =P_{m, n} \mu\left(R^{m}\right) .
\end{aligned}
$$

Next, we prove that $\operatorname{IM}(u) \geq P_{m, n} \mu\left(R^{m}\right)$ when $\mu$ is absolutely continuous with respect to the Lebesgue measure of $R^{m}$. But first, we recall necessary facts about the derivative of such $\mu$.

4.2. Approximation of $u^{*}=f=d \mu / d z$ by Step Functions. Let $\mathbf{z} \in R^{m}$. Denote by $Q(\mathbf{z}, h)$ a cube of side $2 h$ and by $B(\mathbf{z}, h)$ a ball of radius $h$ both centered at $\mathbf{z}$. Let $V_{m}=|B(0,1)|$. When a cube $Q(0, \rho)$ is broken into $N^{m}$ equal cubes $Q_{i}$ of side $l=$ $2 \rho / N$, the smaller cubes of side $l-2 \sigma$ at the centers of $Q_{i}$ are denoted by $\Delta_{i}$.
Lemma 6. Let $f \in L^{1}\left(R^{m}\right), f \geq 0$, and a positive $\varepsilon \ll\|f\|_{1}$ is given. For sufficiently large $\rho$ and $N$, there exist positive numbers $\left\{f_{i}\right\}_{i=1}^{N^{m}}, \sigma$, and $K$ and sets $F, H \subset F$ such that

$$
\left.f\right|_{F \cap Q_{i}} \geq f_{i}
$$

$$
\begin{array}{r}
|B(\mathbf{z}, h) \cap F| \geq|B(\mathbf{z}, h)|\left(1-\frac{\sigma^{m+1}}{V_{m}}\right), \\
\mathbf{z} \in H, \quad h \leq \frac{1}{K},
\end{array}
$$

$$
\sum_{i=1}^{N^{m}} \int_{H \cap \Delta_{i}} f_{i} d \mathbf{z} \geq\|f\|_{1}-5 \varepsilon .
$$

Proof. If the support set, $E$, of $f$ is unbounded, there exists $\rho>0$ such that the integral over the bounded set, $E_{\rho}=E \cap$ $Q(0, \rho)$, satisfies

$$
\int_{E_{\rho}} f(\mathbf{z}) d \mathbf{z}>\|f\|_{1}-\varepsilon
$$

Let $\varepsilon_{j} \downarrow 0, \varepsilon_{1}<\varepsilon$. By Luzin's theorem, there exists a sequence of closed sets $F_{j-1} \subset F_{j} \subset E_{\rho}$ approaching $E_{\rho}$, so that $\left|F_{j}\right| \geq$ $\left|E_{\rho}\right|-\varepsilon_{j}$ and the function $f$ is (uniformly) continuous on $F_{j}$. Let $F=F_{j}$ for $j$ such that

$$
\int_{F} f(\mathbf{z}) d \mathbf{z} \geq \int_{E_{\rho}} f(\mathbf{z}) d \mathbf{z}-\varepsilon \stackrel{(36)}{\geq}\|f\|_{1}-2 \varepsilon .
$$

Now, find $\xi=\xi(\varepsilon, \rho, F)$ so that, for all $\mathbf{z}, \mathbf{w} \in F$, if $|\mathbf{z}-\mathbf{w}|<\xi$, then

$$
|f(\mathbf{z})-f(\mathbf{w})|<\frac{\varepsilon}{(2 \rho)^{m}} .
$$

We break $Q(0, \rho)$ into $N^{m}$ equal cubes $Q_{i}$ with diameters less than $\xi$ taking $N \geq 2 \rho \sqrt{m} / \xi$. Then, (38) implies that, for $\mathbf{z} \in$ $Q_{i}$,

$$
f_{i}=\min _{\mathbf{w} \in F \cap Q_{i}} f(\mathbf{w})>f(\mathbf{z})-\frac{\varepsilon}{(2 \rho)^{m}} .
$$

We observe next that, since $\left|Q_{i} \backslash \Delta_{i}\right| \rightarrow 0$ as $\sigma \rightarrow 0$, there exits $\sigma>0$ small enough to yield

$$
\sum_{i=1}^{N^{m}} \int_{Q_{i} \backslash \Delta_{i}} f(\mathbf{z}) d \mathbf{z}<\varepsilon .
$$

Let $D$ be the set of points of density for $F$. Lebesgue's Theorem states that $|D|=|F|$. Therefore, for $k=1,2, \ldots$, the sets

$$
\begin{aligned}
& D_{k} \\
& =\left\{\mathbf{z} \in D:|B(\mathbf{z}, h) \cap F| \geq|B(\mathbf{z}, h)|\left(1-\frac{\sigma^{m+1}}{V_{m}}\right), h \leq \frac{1}{k}\right\}
\end{aligned}
$$

approach $D$ as $k \rightarrow \infty$. Then, (41) and (37) imply that there exists $K$ such that, for all $k \geq K$,

$$
\int_{D_{k}} f d \mathbf{z} \geq \int_{D} f d \mathbf{z}-\varepsilon=\int_{F} f d \mathbf{z}-\varepsilon \stackrel{(37)}{\geq}\|f\|_{1}-3 \varepsilon
$$


This allows us to take $H=D_{K}$ in (34). Next, we apply (39) to each term in the sum:

$$
\begin{aligned}
\sum_{i} \int_{H \cap \Delta_{i}} f_{i} d \mathbf{z}> & \sum_{i} \int_{H \cap \Delta_{i}} f(\mathbf{z})-\frac{\varepsilon}{(2 \rho)^{m}} d \mathbf{z} \\
= & \sum_{i} \int_{H \cap Q_{i}} f(\mathbf{z}) d \mathbf{z}-\sum_{i} \int_{H \cap\left(Q_{i} \backslash \Delta_{i}\right)} f(\mathbf{z}) d \mathbf{z} \\
& -\sum_{i} \int_{H \cap \Delta_{i}} \frac{\varepsilon}{(2 \rho)^{m}} d \mathbf{z} .
\end{aligned}
$$

It can be seen that $\cup_{i}\left(H \cap Q_{i}\right)=H$ and $H \cap\left(Q_{i} \backslash \Delta_{i}\right) \subset Q_{i} \backslash \Delta_{i}$, and also $\cup_{i}\left(H \cap \Delta_{i}\right) \subset \cup_{i} Q_{i}=Q(0, \rho)$. Hence,

$$
\begin{aligned}
\sum_{i} \int_{H \cap \Delta_{i}} f_{i} d \mathbf{z}> & \int_{H} f(\mathbf{z}) d \mathbf{z}-\sum_{i} \int_{Q_{i} \backslash \Delta_{i}} f(\mathbf{z}) d \mathbf{z} \\
& -\frac{\varepsilon}{(2 \rho)^{m}}|Q(0, \rho)| .
\end{aligned}
$$

The last inequality becomes (35) when (42) (with $H=D_{K}$ ) and (40) are applied.

\subsection{Equality in (4) for Absolutely Continuous $\mu$}

Theorem 7. Let $\Omega$ be the upper half-space $R_{+}^{n+1}$. Let a harmonic function $u$ be defined on $\Omega$ by a finite positive Borel boundary measure $\mu$ that lies on a subspace $R^{m} \subset \partial \Omega$ and is absolutely continuous with respect to the Lebesgue measure of $R^{m}$. Then,

$$
I M(u)=P_{m, n} \mu\left(R^{m}\right)
$$

Proof. By hypothesis, $d \mu(p)=f\left(\mathbf{z}_{p}\right) d \mathbf{z}_{p}$, where $f \in L^{1}\left(R^{m}\right)$, $f \geq 0$. Given $\varepsilon>0$, we obtain constants $\rho, N,\left\{f_{i}\right\}_{i=1}^{N^{m}}, K, \sigma$, sets $F, H$, and cubes $Q_{i} \supset \Delta_{i}$ of sides $l=2 \rho / N, l-2 \sigma$ from Lemma 6. We may assume that $\sigma<\varepsilon$. Let $\delta=\sigma^{4} / K^{2}$. In view of Theorem 5 , it is enough to show that $\int_{\Gamma} u>P_{m, n}\|f\|_{1}-O(\varepsilon)$ for any continuous, piecewise smooth, orientable surface $\Gamma$ in $A_{\delta}$ that separates boundaries of $A_{\delta}$. Consider

$$
\cup \Gamma_{i}=\cup \Gamma \cap\left\{q=(r, \phi, \mathbf{z}): \mathbf{z} \in \Delta_{i} \cap H, r \leq \sqrt{\delta}=\frac{\sigma^{2}}{K}\right\} .
$$

Since the range of $\phi_{1}$ on $\Gamma$ is $[0, \pi / 2)$, the range of $\phi_{1}$ on $\Gamma_{i}$ is $[0, \arccos \sqrt{\delta}]$. When $\mathbf{z} \in \Delta_{i} \cap H$ is fixed, the ball $B(\mathbf{z}, r / \sigma) \subset$ $Q_{i}$ because $r / \sigma \leq \sigma / K$. When $p=\left(0, \mathbf{0}, \mathbf{z}_{p}\right)$ with $\mathbf{z}_{p} \in B\left(\mathbf{z}, \frac{r}{\sigma}\right)$, we have $f\left(\mathbf{z}_{p}\right)>f_{i}$ by (33). Inequality (34) implies that $|B(\mathbf{z}, r / \sigma) \cap F| \geq|B(\mathbf{z}, r / \sigma)|\left(1-\sigma^{m+1} / V_{m}\right)$; that is,

$$
\left|B\left(\mathbf{z}, \frac{r}{\sigma}\right) \backslash F\right| \leq\left|B\left(\mathbf{z}, \frac{r}{\sigma}\right)\right| \frac{\sigma^{m+1}}{V_{m}}=r^{m} \sigma .
$$

Let $q=(r, \phi, \mathbf{z}) \in \Gamma_{i}$; then $|q-p|=\sqrt{r^{2}+\left|\mathbf{z}-\mathbf{z}_{p}\right|^{2}}$, so we may write

$$
\begin{aligned}
\int_{\Gamma} u(q) d \Gamma(q)= & \int_{\Gamma} \int_{R^{m}} \frac{2 y d \mu\left(\mathbf{z}_{p}\right) d \Gamma(q)}{\omega_{n}\left(r^{2}+\left|\mathbf{z}-\mathbf{z}_{p}\right|^{2}\right)^{(n+1) / 2}} \\
> & \frac{2}{\omega_{n}} \sum_{i=1}^{N^{m}} \int_{\Gamma_{i}} \frac{y / r d \Gamma(q)}{r^{n-m}} f_{i} \\
& \times \int_{B(z, r / \sigma) \cap F} \frac{d \mathbf{z}_{p} / r^{m}}{\left(1+\left|\mathbf{z}-\mathbf{z}_{p}\right|^{2} / r^{2}\right)^{(n+1) / 2}}
\end{aligned}
$$

The change of variables $\mathbf{v}=\left(\mathbf{z}_{p}-\mathbf{z}\right) / r$ defines a map $T$ : $B(\mathbf{z}, r / \sigma) \rightarrow B(0,1 / \sigma)$ and a set $\Upsilon=T(B(\mathbf{z}, r / \sigma) \backslash F)$ whose size $|\Upsilon| \leq r^{m} \sigma\left(1 / r^{m}\right)=\sigma$ by (47). Now, Lemma 3 estimates the factor to the right of $f_{i}$ in (48) as follows:

$$
\begin{aligned}
& \int_{B(\mathbf{z}, r / \sigma) \cap F} \frac{d \mathbf{z}_{p} / r^{m}}{\left(1+\left|\mathbf{z}_{p}-\mathbf{z}\right|^{2} / r^{2}\right)^{(n+1) / 2}} \\
& \quad=\int_{B(0,1 / \sigma) \backslash \Upsilon} \frac{d \mathbf{v}}{\left(1+|\mathbf{v}|^{2}\right)^{(n+1) / 2}}>G_{m, n}-35 \sigma .
\end{aligned}
$$

If $\Gamma$ is given by $r=r(\phi, \mathbf{z})$, we have $d \Gamma(r, \phi, \mathbf{z}) \geq$ $r^{n-m} d S^{n-m}(\phi) d \mathbf{z}$ by Lemma 2. Then, (35) and (22) estimate the sum:

$$
\begin{aligned}
& \sum_{i} \int_{\Gamma_{i}} \frac{y d \Gamma(q)}{r r^{n-m}} f_{i} \\
& \quad>\int_{S_{+}^{n-m} \cap\left\{\cos \phi_{1} \geq \sqrt{\delta}\right\}} \frac{y}{r} d S^{n-m}(\phi) \sum_{i} \int_{\Delta_{i} \cap H} f_{i} d \mathbf{z} \\
& \quad>\left(\int_{S_{+}^{n-m}} \frac{y}{r} d S^{n-m}-\eta\right)\left(\|f\|_{1}-5 \varepsilon\right) \\
& \quad=\left(S_{m, n}-\eta\right)\left(\|f\|_{1}-5 \varepsilon\right),
\end{aligned}
$$

where $\eta=0$ if $m=n$, and $\eta=\omega_{n-m-1}\left[1-(1-\delta)^{(n-m) / 2}\right] /(n-$ $m)=O(\delta)=o(\varepsilon)$ otherwise. Finally, (48)-(50) and Lemma 4 yield the desired inequality:

$$
\begin{aligned}
\int_{\Gamma} u d \Gamma & >\frac{2}{\omega_{n}}\left(S_{m, n}-\eta\right)\left(\|f\|_{1}-5 \varepsilon\right)\left(G_{m, n}-35 \sigma\right) \\
& =P_{m, n}\|f\|_{1}-\zeta,
\end{aligned}
$$

with $\zeta=\left(2 / \omega_{n}\right)\left(5 \varepsilon S_{m, n}+\eta\|f\|_{1}-5 \eta \varepsilon\right)\left(G_{m, n}-35 \sigma\right)=O(\varepsilon)$.

Since $\varepsilon, \Gamma$ were arbitrary and $\|f\|_{1}=\mu\left(R^{m}\right)$, the inferior mean satisfies

$$
\operatorname{IM}(u)=\lim _{\delta \rightarrow 0} \inf _{\Gamma \subset A_{\delta}} \int_{\Gamma} u(q) d \Gamma(q) \geq P_{m, n} \mu\left(R^{m}\right) .
$$

The opposite inequality of Theorem 5 gives us equality in (52). 


\section{Measures on Curves}

In this section, we show that if $u$ is defined by a boundary measure $\mu$ lying on a smooth curve, $\gamma$, then $\operatorname{IM}(u)$ is bounded by the integrals of $u(q)$ over half-tubes $\{q \in \Omega$ : $\operatorname{dist}(q, \gamma)=$ $r$ \} about $\gamma$. If $\mu$ is the arclength measure of $\gamma$, these integrals approach $\operatorname{IM}(u)$ as $r \rightarrow 0$. We assume that the dimension $n$ of $\partial \Omega=R^{n}$ is greater than 1 . The case $n=1$, where $\gamma=R^{1}$ or $\gamma=[a, b]$, is covered by Theorems 5 and 7 .

5.1. On Linear Approximation of $C^{1}$-Curves. Let $\gamma:[0,1] \rightarrow$ $R^{n}$ be a simple $C^{1}$-curve parameterized by arclength. Let $\tau$ be $\gamma$ 's injectivity radius (radius of the largest embedded normal tube around $\gamma$ ).

Lemma 8. For any $\varepsilon>0$, there exists $v<\min \{\tau, \varepsilon\}$ so that, for $|s-t|<\nu$, one has the following:

$$
\begin{gathered}
|\gamma(s)-\gamma(t)| \leq|s-t| \leq \frac{|\gamma(s)-\gamma(t)|}{(1-\varepsilon)}, \\
\max _{\xi \in(t, s)} \operatorname{dist}(\gamma(\xi), \gamma(t)-\gamma(s))<\varepsilon|s-t| .
\end{gathered}
$$

For any $q \in R_{+} \times R^{n}$, there exists $t_{q}$ such that

$$
r_{q}=\operatorname{dist}(q, \gamma)=\left|q-\gamma\left(t_{q}\right)\right| \text {. }
$$

If $t_{q} \neq 0,1$ and $q$ is sufficiently close to $\gamma$, then

$$
|q-\gamma(s)|<\sqrt{r_{q}^{2}+\left(s-t_{q}\right)^{2}}(1+\varepsilon) .
$$

Proof. Since $\gamma^{\prime}$ is continuous, there exists $\nu$ such that, for $s, t \in$ $[0,1]:|s-t|<\nu$, there exist $n$ numbers $\beta_{i} \in(t, s), i=1, \ldots, n$, so that

$$
\begin{gathered}
\gamma_{i}(s)-\gamma_{i}(t)=(s-t) \gamma_{i}^{\prime}\left(\beta_{i}\right), \\
\left|\gamma_{i}^{\prime}\left(\beta_{i}\right)-\gamma_{i}^{\prime}(t)\right|<\frac{\varepsilon}{3 \sqrt{n}} .
\end{gathered}
$$

This implies (53) because we have length $\left(\left.\gamma\right|_{[t, s]}\right)=|s-t|$. Denote by $\mathbf{d}$ the unit vector $\gamma^{\prime}(t)$. Using (57), we write vectors $\mathbf{w}, \mathbf{u}$ below as

$$
\begin{aligned}
& \mathbf{w}=\gamma(s)-\gamma(t)=(s-t)(\mathbf{d}+\mathbf{e}), \\
& \mathbf{u}=\gamma(\xi)-\gamma(t)=(\xi-t)(\mathbf{d}+\mathbf{h}),
\end{aligned}
$$

where $|\mathbf{e}|,|\mathbf{h}|<\varepsilon / 3$. Then, (54) follows from the estimates of $\angle \mathbf{w}, \mathbf{u}=A$ :

$$
\begin{aligned}
& \sin ^{2} A=1-\frac{(\mathbf{w} \cdot \mathbf{u})^{2}}{(\mathbf{w} \cdot \mathbf{w})(\mathbf{u} \cdot \mathbf{u})} \\
&=\mid(\mathbf{e} \cdot \mathbf{e}+\mathbf{h} \cdot \mathbf{h}+2(\mathbf{d} \cdot \mathbf{e})(\mathbf{d} \cdot \mathbf{h}) \\
&\left.\quad-(\mathbf{d} \cdot \mathbf{e})^{2}-(\mathbf{d} \cdot \mathbf{h})^{2}-2(\mathbf{e} \cdot \mathbf{h})+\sigma\right) \\
& \quad \times((1+2 \mathbf{d} \cdot \mathbf{e}+\mathbf{e} \cdot \mathbf{e})(1+2 \mathbf{d} \cdot \mathbf{h}+\mathbf{h} \cdot \mathbf{h}))^{-1} \mid \\
&<\varepsilon^{2} .
\end{aligned}
$$

One can check that $\sigma<\varepsilon^{3}$. Let $q, r_{q}$, and $t_{q}$ be as in (55) and $\mathbf{r}_{q}=q-\gamma\left(t_{q}\right)$. If $t_{q} \neq 0,1$, then $\mathbf{r}_{q} \perp \mathbf{d}$. Now, (56) is obtained from

$$
\begin{aligned}
|q-\gamma(s)|^{2}= & \left|q-\gamma\left(t_{q}\right)+\gamma\left(t_{q}\right)-\gamma(s)\right|^{2}=\left|\mathbf{r}_{q}-\mathbf{w}\right|^{2} \\
= & \mathbf{r}_{q} \cdot \mathbf{r}_{q}+\left(s-t_{q}\right)^{2}(1+2 \mathbf{d} \cdot \mathbf{e}+\mathbf{e} \cdot \mathbf{e}) \\
& -2\left(s-t_{q}\right) \mathbf{r}_{q} \cdot \mathbf{e} .
\end{aligned}
$$

5.2. Inequality (4) for Measures on Curves. Since measures $\mu$ are finite, we may assume that curves are bounded (see (36)).

Theorem 9. Let $\Omega$ be the upper half-space $R_{+}^{n+1}$ and let $\gamma$ : $[0,1] \rightarrow \partial \Omega$ be a simple $C^{1}$-curve. Let a harmonic function $u$ be defined on $\Omega$ by a finite positive Borel boundary measure $\mu$ that lies on $\gamma$. Then,

$$
I M(u) \leq P_{1, n} \mu(\gamma) .
$$

Proof. We assume that $\gamma$ is parameterized by the arclength parameter $s$. Thus, $|\gamma|=1$. Let $0<\varepsilon<10^{-4}$. Consider a curve $\gamma$ with injectivity radius $\tau$ and $\nu=\nu(\gamma, \varepsilon, \tau)$ as in Lemma 8. We will show that, for $\delta=\nu^{2} \varepsilon^{1 / 2}$, there exists a surface $\Gamma \subset A_{\delta}$ such that

$$
\int_{\Gamma} u(q) d \Gamma<P_{1, n} \mu(\gamma)+O\left(\varepsilon^{1 / 2}\right) .
$$

Let $\left.[0,1]=\cup_{i=1}^{N}[(i-1) / N), i / N\right]$ and let $\cup_{i=1}^{N} l_{i}$ be a polygonal approximation of $\gamma$ with line segments $l_{i}$ connecting $\gamma((i-$ $1) / N)$ with $\gamma(i / N)$. We take

$$
N=\left[\left[\frac{2}{v^{2}}\right]\right]+1, \quad l=\frac{1}{N}, \quad r=l \varepsilon^{1 / 2} .
$$

Note that $\sqrt{l}<v$ and $r<\delta$. It follows from (53) and (54) that

$$
\max _{s} \operatorname{dist}\left(\gamma(s), \bigcup_{i=1}^{N} l_{i}\right)<l \varepsilon \ll r \ll l(1-\varepsilon)<\left|l_{i}\right| \leq l .
$$

Let $l_{0}$ and $l_{N+1}$ be segments of length $l$ extending $l_{1}$ and $l_{N}$. Denote by $L_{i}$ lines containing $l_{i}$. We build a Quonset hut $\Gamma_{T}=$ $\cup \Gamma_{i}$ above $\gamma$ with the axis along $\cup l_{i}$ planted in the hyperplane $H=\left\{q \in \Omega: y=(r / 2)^{2}\right\}$ (See Figure 1). It consists of $N+$ 2 straight cylindrical huts $\Gamma_{i} \subset T_{r}^{1}\left(L_{i}\right)$ glued together where $T_{r}^{1}\left(L_{i}\right)$ meets $T_{r}^{1}\left(L_{i \pm 1}\right)$ and two spherical doors $\Gamma_{-1}$ and $\Gamma_{N+2}$. The part of $H$ outside the hut is called $\Gamma_{H}$ and our surface $\Gamma$ is $\Gamma_{T} \cup \Gamma_{H}$. By (64), $\Gamma_{H} \subset H_{r / 2}^{\mu}$ (subset of $H$ is defined in (28)). Thus by (29),

$$
\int_{\Gamma_{H}} u d H<\int_{H_{r / 2}^{\mu}} u d H=O\left(\frac{r}{2}\right)=o(\varepsilon) .
$$

We apply Fubini's theorem to $\int_{\Gamma_{T}} u$ and estimate $\int_{\Gamma_{T}} P(q, \gamma(s))$ for a fixed $s \in[(i-1) / N, i / N]$. The main contribution to $\int_{\Gamma_{T}} P$ will come from $\Gamma_{i} \cup \Gamma_{i \pm 1}$. 

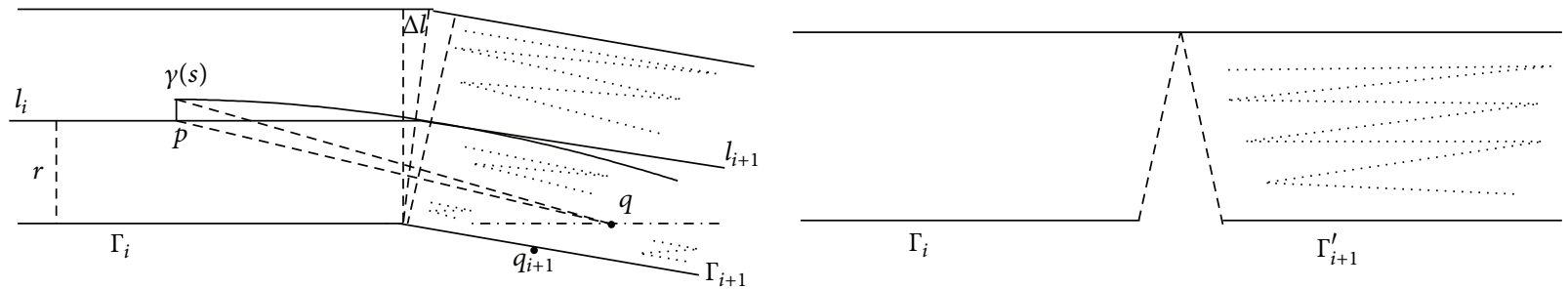

$r=l \varepsilon^{1 / 2},\left|l_{i}\right| \approx l, \Delta l<2 r \varepsilon$, length $\left(\Gamma_{i}\right) \leq l+2 \Delta l, \sin \angle l_{i}, l_{i+1}<2 \varepsilon$ by (59).

Figure 1

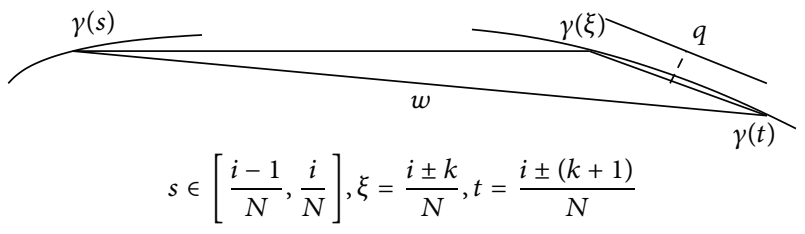

Figure 2

Integration over $\Gamma_{i+1}$ (and similarly $\Gamma_{i-1}$ ) is replaced with that over its copy, $\Gamma_{i+1}^{\prime}$, on $T_{r}^{1}\left(L_{i}\right)$ obtained by swiveling $\Gamma_{i+1}$ to $\Gamma_{i}$ until the two contours are aligned horizontally (see Figure 1). Under this rotation, the distance of $q_{i+1} \in \Gamma_{i+1}$ to $\partial \Omega$ is preserved and the distance to its image $q \in \Gamma_{i+1}^{\prime}$ is

$$
\left|q_{i+1}-q\right|<(l+2 \Delta l) \sin \angle l_{i}, l_{i+1}+2 \Delta l<3 l \varepsilon .
$$

If $p$ is the projection of $\gamma(s)$ on $l_{i}$, then, by (64), $|p-\gamma(s)|<l \varepsilon$. So

$$
\begin{aligned}
\left|q_{i+1}-\gamma(s)\right| & >|q-p|-|p-\gamma(s)|-\left|q_{i+1}-q\right| \\
& >|q-p|\left(1-4 \varepsilon^{1 / 2}\right) .
\end{aligned}
$$

That gives us $P\left(q_{i+1}, p\right)<P(q, p) /\left(1-4 \varepsilon^{1 / 2}\right)^{n+1}$ and now, with the assumption that $\gamma(s)$ is closer to $\Gamma_{i+1}$, Lemma 4 yields the main estimate:

$$
\begin{aligned}
\int_{\Gamma_{i} \cup \Gamma_{i \pm 1}} P d \Gamma_{T} & <\int_{\Gamma_{i}} P(q, \gamma(s)) d \Gamma_{i}+2 \int_{\Gamma_{i+1}} P\left(q_{i+1}, \gamma(s)\right) d \Gamma_{i+1} \\
& <\frac{1}{\left(1-4 \varepsilon^{1 / 2}\right)^{n+1}} \int_{T_{r}^{1}\left(L_{i}\right)} P(q, p) d T_{r}^{1}(q) \\
& =P_{1, n}+O\left(\varepsilon^{1 / 2}\right) .
\end{aligned}
$$

Next, we integrate over $\Gamma_{i \pm k}, k \geq 2$ as far as $|q-\gamma(s)|<\sqrt{l}<$ $v$ (where (53)-(59) of Lemma 8 are valid). Let $s, \xi, t$ be as in Figure 2.

By (59), the angle $\angle \gamma(s) \gamma(\xi) \gamma(t)>\pi-2 \varepsilon$, so $\gamma(s)$ is closer to $\gamma(\xi)$ than to the projection of $q \in \Gamma_{i \pm k}$ on $l_{i \pm k}$. But $\mid \gamma(s)-$ $\gamma(\xi)|>| s-\xi \mid(1-\varepsilon)$ by (53). Hence, by the triangle inequality, we have a lower bound for

$$
\begin{aligned}
|\gamma(s)-q| & >|\gamma(s)-\gamma(\xi)|-r \\
& >|s-\xi|(1-\varepsilon)-r \geq \frac{k-1}{N}(1-\varepsilon)-r \\
& =(k-1) l\left(1-\varepsilon-\frac{\sqrt{\varepsilon}}{k-1}\right)>(k-1) \frac{l}{2} .
\end{aligned}
$$

Recall that $n \geq 2, r=l \varepsilon^{1 / 2}, y=\operatorname{dist}(q, \partial \Omega) \leq r,\left|\Gamma_{k}\right|<$ $2 l r^{n-1} \omega_{n-1}$ and $\left|\Gamma_{T}\right|<2 r^{n-1} \omega_{n-1}$. These, combined with (69), estimate the integrals of $P$ over the two remaining parts of $\Gamma_{T}$ as follows:

$$
\begin{aligned}
& \sum_{\substack{k \geq 2 \\
|q-\gamma(s)|<\sqrt{l}}} \int_{\Gamma_{i \pm k}} \frac{2 y / \omega_{n} d \Gamma_{T}}{|q-\gamma(s)|^{n+1}} \\
& <\frac{2}{\omega_{n}} \sum_{k \geq 2} \frac{2 r 2 l r^{n-1} \omega_{n-1}}{((k-1) l / 2]^{n+1}}=O\left(\varepsilon^{n / 2}\right), \\
& \int_{\Gamma_{T} \cap\{\{q-\gamma(s) \mid \geq \sqrt{l}\}} \frac{2 y / \omega_{n} d \Gamma_{T}}{|q-\gamma(s)|^{n+1}} \\
& <\frac{2 r 2 r^{n-1} \omega_{n-1}}{\omega_{n} l^{(n+1) / 2}}=O\left(l^{(n-1) / 2} \varepsilon^{n / 2}\right) .
\end{aligned}
$$

Finally, from (65), (68), (70), and Fubini's theorem, we can obtain the estimate (62):

$$
\begin{aligned}
\int_{\Gamma_{H} \cup \Gamma_{T}} u d \Gamma & =o(\varepsilon)+\int_{0}^{1} \int_{\Gamma_{T}} P(q, \gamma(s)) d \Gamma_{T}(q) d \mu(\gamma(s)) \\
& =o(\varepsilon)+\int_{0}^{1} P_{1, n}+O\left(\varepsilon^{1 / 2}\right) d \mu(\gamma(s)) \\
& =P_{1, n} \mu(\gamma)+O\left(\varepsilon^{1 / 2}\right) .
\end{aligned}
$$

\subsection{Equality in (4) for Arclength Measures}

Theorem 10. Let $\Omega$ be the upper half-space $R_{+}^{n+1}$ and let $\gamma$ : $[0,1] \rightarrow \partial \Omega$ be a simple $C^{1}$-curve. Let a harmonic function 


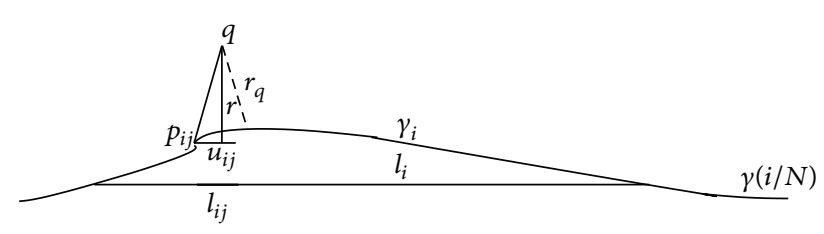

Figure 3

$u$ be defined on $\Omega$ by the boundary measure $\mu$, that is, the arclength measure of $\gamma$. Then,

$$
I M(u)=P_{1, n} \mu(\gamma) .
$$

Proof. We assume that $\gamma$ is parameterized by the arclength parameter $s$. Thus, $\mu(\gamma)=|\gamma|=1$ and $d \mu(\gamma(s))=d s$. Let $0<\varepsilon<10^{-4}$. Consider a curve $\gamma$ with injectivity radius $\tau$ and $\nu=\nu(\gamma, \varepsilon, \tau)$ as in Lemma 8. We fix an integer $N>1 / \nu$, and, for $i=1, \ldots, N$, define

$$
\begin{gathered}
\sigma=\frac{\varepsilon}{2 N}, \quad \delta=\sigma^{4}, \quad \Delta_{i}=\left[\frac{i-1}{N}+\sigma, \frac{i}{N}-\sigma\right], \\
\gamma_{i}=\left.\gamma\right|_{\Delta_{i}} .
\end{gathered}
$$

Note that $\sigma \ll 1 / N<v<\tau$. Consider segments $l_{i}$ connecting endpoints of $\gamma_{i}$; then, by (54) and (73),

$$
\sum_{i}\left|l_{i}\right|>\sum_{i}\left|\Delta_{i}\right|(1-\varepsilon)=(1-\varepsilon)^{2} .
$$

In view of Theorem 9, it suffices to show that, for any continuous piecewise smooth surface $\Gamma$ in $A_{\delta}$ separating its boundaries,

$$
\int_{\Gamma} u d \Gamma>P_{1, n}-O(\varepsilon)
$$

The $\operatorname{dist}(\Gamma, \gamma)$ can be quite small; therefore, we break $l_{i}$ into equal segments $l_{i j}$ and place their copies, $u_{i j}$, at points $p_{i j}$ on $\gamma_{i}$ so that $l_{i j}, u_{i j}$ are parallel sides in rectangles (Figure 3 ). We require that

$$
\left|l_{i j}\right|=\left|u_{i j}\right| \leq[\operatorname{dist}(\Gamma, \gamma)]^{2} .
$$

In local tubular coordinates where the short $z$-axes lie on $u_{i j}$, let

$$
\Gamma_{i j}=\left\{q=(r, \phi, z) \in \Gamma: z \in u_{i j}, r=\operatorname{dist}\left(q, u_{i j}\right) \leq \sqrt{\delta}\right\} .
$$

Fix $q \in \Gamma_{i j}$. Recall that $r_{q}=\operatorname{dist}(q, \gamma)=\left|q-t_{q}\right|$. Then, (76) yields

$$
\begin{gathered}
\left|u_{i j}\right|^{1 / 2} \leq r_{q} \leq \sigma^{2} \Longrightarrow r_{q}-\left|u_{i j}\right| \geq r_{q}-r_{q}^{2}>r_{q}\left(1-\sigma^{2}\right), \\
r \geq\left|q-p_{i j}\right|-\left|u_{i j}\right| \geq r_{q}-\left|u_{i j}\right| \geq r_{q}\left(1-\sigma^{2}\right) .
\end{gathered}
$$

Next, we claim that $\Gamma_{i}=\cup_{j} \Gamma_{i j}$ (and thus $\Gamma_{i j}$ ) do not overlap. Indeed,

$$
\begin{gathered}
\operatorname{dist}\left(\gamma_{i}, \gamma_{k}\right) \geq 2 \tau, \quad k \neq i \pm 1, \quad \operatorname{dist}\left(\gamma_{i}, \gamma_{i+1}\right) \approx 2 \sigma>\sigma, \\
\max _{q \in \Gamma_{i}} r_{q}=\max _{j} \max _{q \in \Gamma_{i j}} r_{q} \leq \sqrt{\delta}=\sigma^{2}
\end{gathered}
$$

prove the claim

$$
\operatorname{dist}\left(\Gamma_{i}, \Gamma_{k}\right)>\operatorname{dist}\left(\gamma_{i}, \gamma_{k}\right)-\max _{q \in \Gamma_{i}} r_{q}-\max _{q \in \Gamma_{k}} r_{q}>\sigma-2 \sigma^{2}>0 .
$$

Now, the first estimate below is analogous to (49) of Theorem 7. It is obtained from (56), (80), the change of variables $v=\left(s-t_{q}\right) / r_{q}$, and Lemma 3 with $\rho=1 / \sigma$ and $\Upsilon=\emptyset$

$$
\begin{aligned}
\int_{\gamma} \frac{d \mu(\gamma(s))}{|q-\gamma(s)|^{n+1}} & >\int_{\Delta_{i}} \frac{d s}{|q-\gamma(s)|^{n+1}} \\
& >\int_{t_{q}-r_{q} / \sigma}^{t_{q}+r_{q} / \sigma} \frac{(1+\varepsilon)^{-n-1} d s}{\left[r_{q}^{2}+\left(s-t_{q}\right)^{2}\right]^{(n+1) / 2}} \\
& =\frac{1}{r_{q}^{n}} \int_{-1 / \sigma}^{1 / \sigma} \frac{(1+\varepsilon)^{-n-1} d v}{\left(1+v^{2}\right)^{(n+1) / 2}} \\
& >\frac{G_{1, n}-34 \sigma}{r_{q}^{n}(1+\varepsilon)^{n+1}} .
\end{aligned}
$$

Since the range of $\phi_{1}$ on $\Gamma$ is $[0, \pi / 2)$, the range of $\phi_{1}$ on $\Gamma_{i j}$ is $[0, \arccos \sqrt{\delta}]$. If $\Gamma$ is given by $r=r(\phi, z)$, then $d \Gamma(r, \phi, z) \geq$ $r^{n-1} d S^{n-1} d z$ by Lemma 2 . The second estimate is analogous to (50) of Theorem 7. It is obtained from (22) and (74) as follows:

$$
\begin{aligned}
\int_{\Gamma} \frac{y d \Gamma}{r_{q}^{n}} & >\int_{\cup_{i, j} \Gamma_{i j}} \frac{y d \Gamma}{r_{q}^{n}} \\
& >\int_{S_{+}^{n-1} \cap\left\{\cos \phi_{1} \geq \sqrt{\delta}\right\}} \frac{y r^{n-1} d S^{n-1}}{r^{n} /\left(1-\sigma^{2}\right)^{n}} \int_{\cup_{i, j} u_{i j}} d z \\
& >\left(S_{1, n}-\eta\right)\left(1-\sigma^{2}\right)^{n}(1-\varepsilon)^{2},
\end{aligned}
$$

where $\eta=\omega_{n-2}\left[1-(1-\delta)^{(n-1) / 2}\right] /(n-1)$ and we estimated $\sum_{i, j}\left|u_{i j}\right|=\sum_{i}\left|l_{i}\right|>(1-\varepsilon)^{2}$ using $(74)$.

Finally, $\sigma, \eta, \delta<\varepsilon$, Lemma 4 , and (82)-(83) yield the inequality (75):

$$
\begin{aligned}
\int_{\Gamma} u d \Gamma & =\int_{\Gamma} \frac{2 y}{\omega_{n}}\left(\int_{\gamma} \frac{d \mu(\gamma(s))}{|q-\gamma(s)|^{n+1}}\right) d \Gamma \\
& >\int_{\Gamma} \frac{2 y}{\omega_{n}} \frac{\left(G_{1, n}-34 \sigma\right)}{r_{q}^{n}(1+\varepsilon)^{n+1}} d \Gamma \\
& >\frac{2}{\omega_{n}} \frac{\left(S_{1, n}-\eta\right)\left(G_{1, n}-34 \sigma\right)\left(1-\sigma^{2}\right)^{n}(1-\varepsilon)^{2}}{(1+\varepsilon)^{n+1}} \\
& =P_{1, n}-O(\varepsilon) .
\end{aligned}
$$




\section{Conjectures}

With methods of Theorems 9 and 10 and triangulation of mdimensional manifolds, we conjecture that these theorems are true for $m \geq 1$.

Conjecture 1. Let $\Omega$ be the upper half-space $R_{+}^{n+1}$. Let a harmonic function $u$ be defined on $\Omega$ by a finite positive Borel boundary measure $\mu$ that lies on an $m$-dimensional manifold $\gamma \subset \partial \Omega, m \in\{0, \ldots, n\}$, and then

$$
I M(u) \leq P_{m, n} \mu(\gamma) .
$$

Moreover, equality in (85) holds, if $\mu$ is the volume measure of $\gamma$.

The next conjecture, if true, combines the results of Theorems 7 and 10.

Conjecture 2. With the assumptions of Conjecture 1, equality in (85) holds, if $\mu$ is absolutely continuous with respect to the volume measure of $\gamma$.

\section{Conflict of Interests}

The author declares that there is no conflict of interests regarding the publication of this paper.

\section{Acknowledgments}

The author is very grateful to P. G. Andrews, D. Berg, C. I. Delman, J. F. Glazebrook, and C. M. Hawker for their assistance.

\section{References}

[1] J. M. Wu, An integral problem for positive harmonic functions [Ph.D thesis], UIUC, 1974.

[2] Y. Movshovich, "Surface integrals and harmonic functions," Journal of Inequalities and Applications, no. 4, pp. 443-448, 2005.

[3] G. M. Fichtenholz, Differential-und Integralerchnung, VEB Deutsher Verlag der Wissenschaften, Berlin, Germany, 1982.

[4] A. Sard, "The measure of the critical values of differentiable maps," Bulletin of the American Mathematical Society, vol. 48, pp. 883-890, 1942.

[5] S. Axler, P. Bourdon, and W. Ramey, Harmonic Function Theory, Springer, New York, NY, USA, 1992.

[6] P. L. Duren, Theory of $\mathrm{H}^{p}$ Spaces, Academic Press, New York, NY, USA, 1970.

[7] E. M. Stein and G. Weiss, Introduction to Fourier Analysis on Euclidean Spaces, Princeton University Press, Princeton, NJ, USA, 1971. 


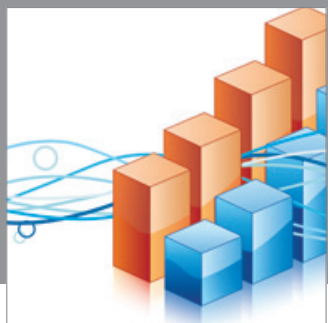

Advances in

Operations Research

mansans

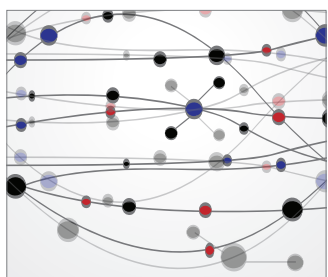

The Scientific World Journal
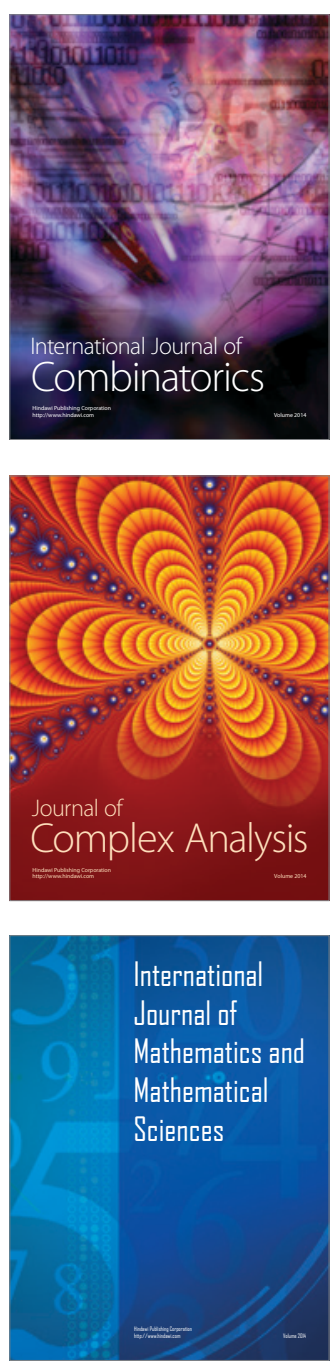
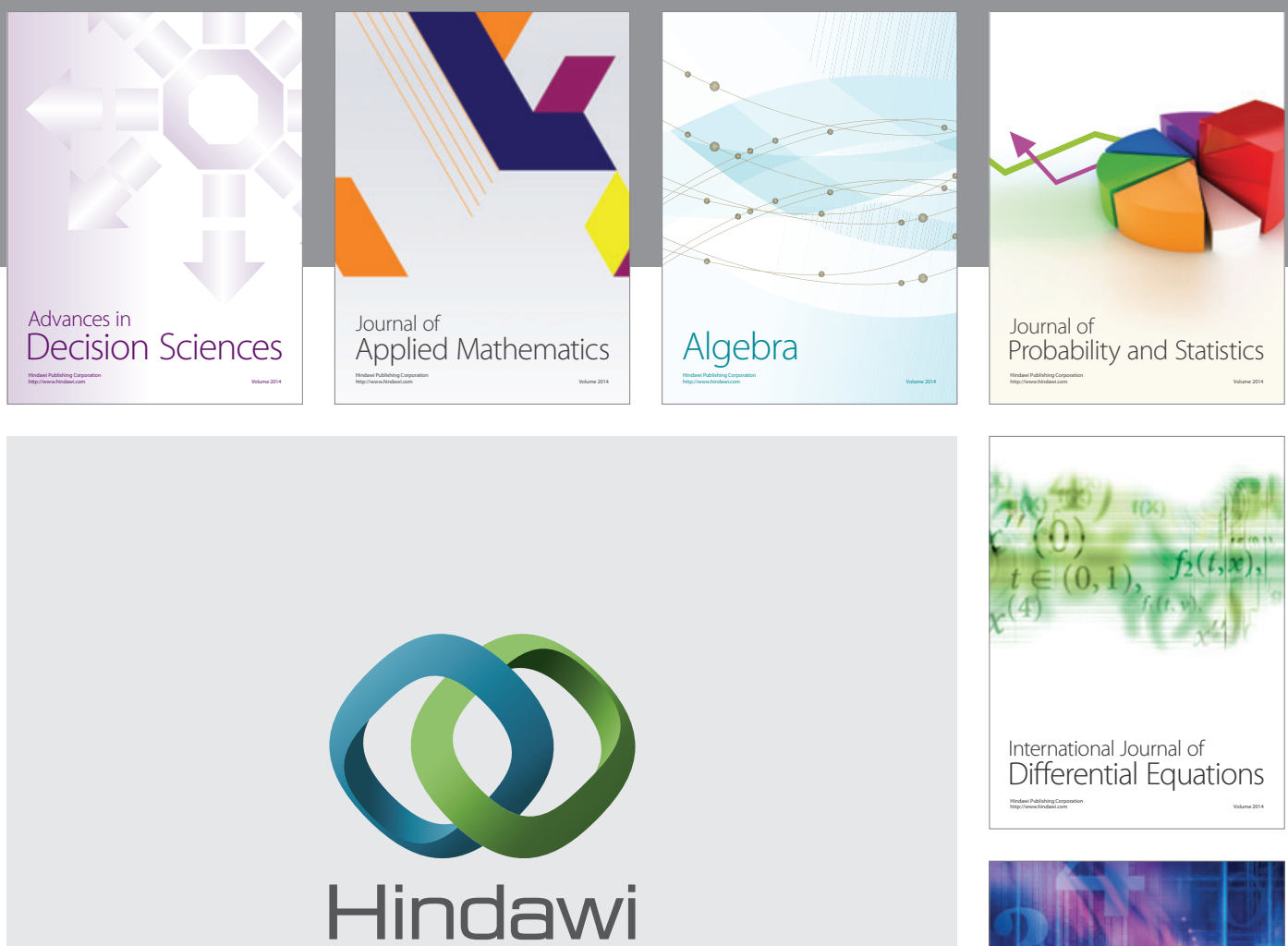

Submit your manuscripts at http://www.hindawi.com
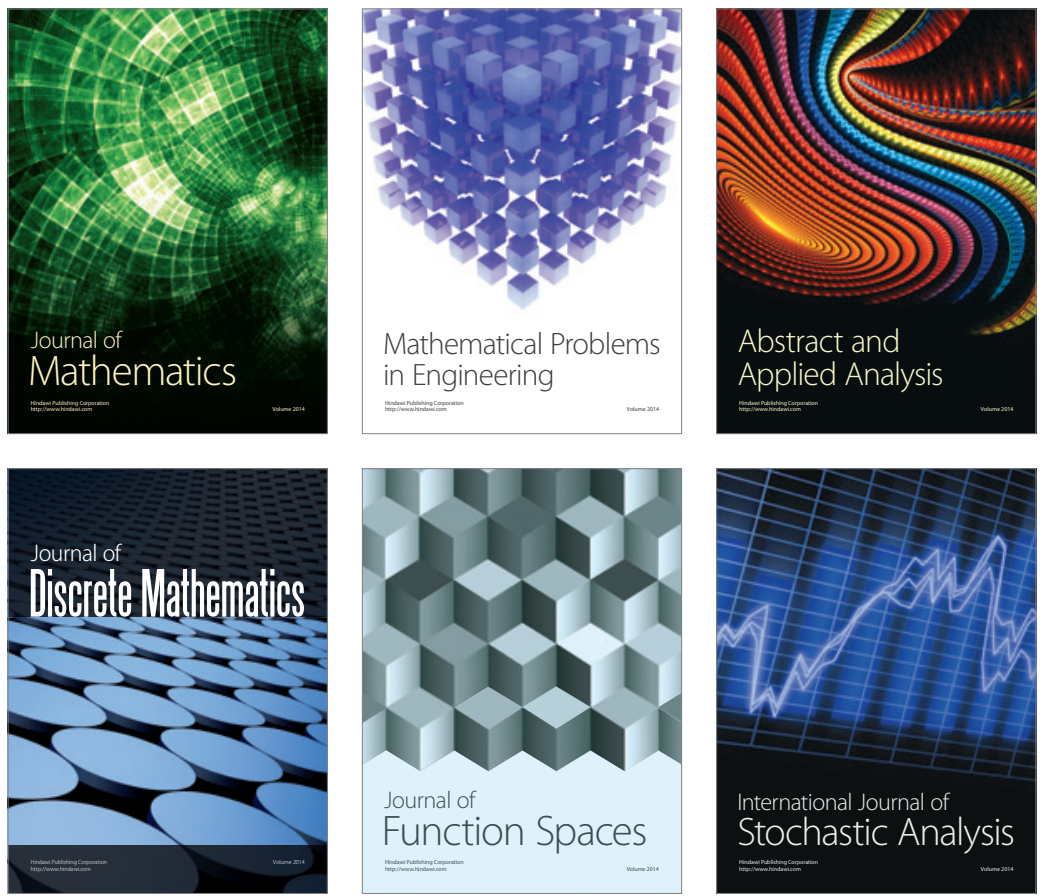

Journal of

Function Spaces

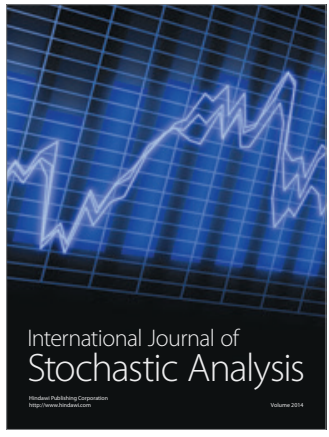

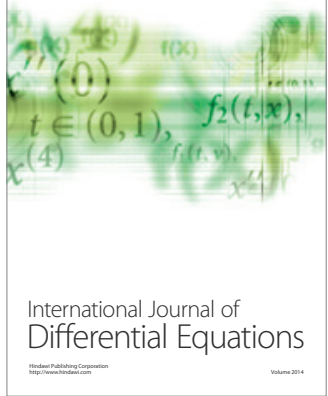
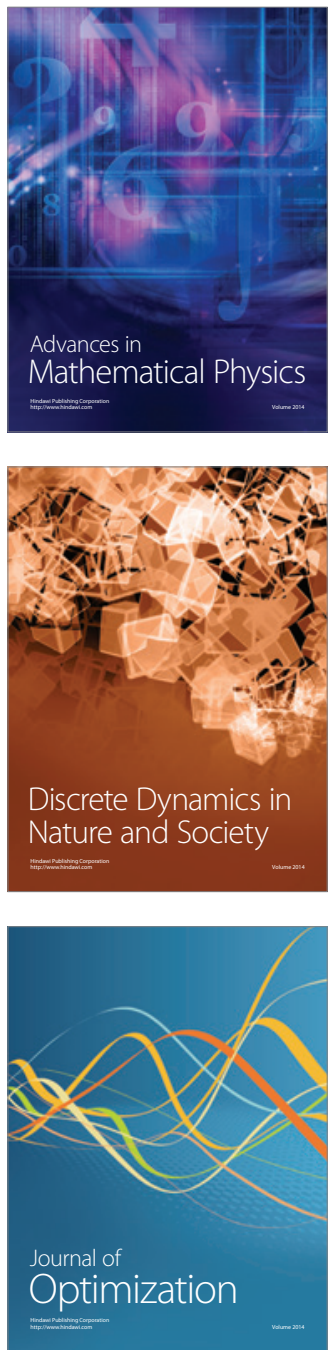